\title{
2.1 Pericope adulterae - Das Ostrakon London, British Museum, EA 21424
}

Matthias H. O. Schulz 

Keine bekannte sahidische Johanneshandschrift bietet die Perikope von Jesus und der Ehebrecherin $(\mathrm{J} 7,53-8,11)$ und auch bohairisch ist sie selten belegt, ${ }^{63}$ doch H. R. Hall veröffentlichte 1905 ein Kalksteinostrakon, dessen Text er als Teil der Perikope identifizierte (J 8,9-11). ${ }^{64}$ O. von Lemm untersuchte den Text 1910 und legte eine um Ergänzungen vervollständigte Fassung vor. ${ }^{65}$ Bedauerlicherweise sind Halls Edition und Umzeichnung des Ostrakon ungenau. Da dies der einzig bekannte Textbeleg ist, seien Neuedition und von Lemms Rekonstruktion mit Kommentar und revidierter Textfassung geboten. ${ }^{66}$

Das Ostrakon ist von heller, beigegrauer Farbe, unvollständig erhalten und weist allseits klar erkennbare Bruchkanten auf. Die Schrift ist dunkelgrau, stellenweise leicht verblasst, doch insgesamt gut erkennbar, und war wohl ursprünglich schwarz. Sehr wahrscheinlich wurde zur Beschriftung Tusche auf Rußbasis verwendet, da keine der üblicherweise erkennbaren rot-bräunlichen Verfärbungen aufgrund von Oxidation feststellbar sind, die etwa Eisengallustinte alterungsbedingt eigen sind. Weder Hall noch von Lemm datieren das Ostrakon. ${ }^{67}$ Trismegistos gibt als Datierungsrahmen „AD 400-899“ an. ${ }^{68}$ Zumal die Schrift teils ungelenk ist als auch wenig charakteristische Merkmale aufweist, die einen sicheren Datierungsvorschlag erlauben, ist eine genauere zeitliche Einordnung kaum möglich. Da allerdings das Vorkommen der pericope adulterae in bohairischen Handschriften und das gänzliche Fehlen ihrer in sahidischen Handschriften auffällig ist, ist der zeitliche Abstand zwischen Ostrakon und bohairischem Text wohl eher kürzer als länger zu vermuten, was einen Hinweis auf ein jüngeres Entstehungsdatum bedeuten könnte.

$63 \mathrm{Zu}$ Text und Handschriften siehe Horner 1898: 420-431.

64 London, BM, EA 21424 (O.Brit.Mus.Copt. I 20:2; TM/LDAP 111700), ediert in Hall 1905: 27 Nr. 2 mit Tf. 20,2; https://www.britishmuseum.org/collection/object/Y_EA21424.

65 Von Lemm 1910: 69-71 (LXIX.9).

66 Nach J. Knust und T. Wassermann habe Ch. Askeland in persönlicher Kommunikation vom 2. Juni 2009 die Identifizierung durch Hall in Zweifel gezogen, da die erhaltenen Wörter und Phrasen (Priester, Frau, „er sagte“) gewöhnlich seien und auch in Privatbriefen Verwendung fänden, siehe Knust/Wassermann 2010: 424 mit Anm. 81. Weder Knust und Wassermann noch Askeland berücksichtigen von Lemms Arbeit und seine philologisch gut begründeten Ergänzungen. Askelands Argumentation bedenkt nicht die Unwahrscheinlichkeit, dass in einem Privatbrief die von ihm genannten Wörter und Phrasen in exakt der notwendigen Abfolge vorkommen und alle anderen Wörter und Wortteile sinnvoll dem Text der Perikope entsprechen. 67 Hall 1905: 27; von Lemm 1910: 69-71.

68 Siehe https://www.trismegistos.org/tm/detail.php?tm=111700. 
London, BM, EA 21424

ca. $9,5 \times 8,0 \mathrm{~cm}$

Kalkstein

Herkunft unbekannt

5.-9. Jh. (TM)

1

].. [

N̄GI Neח]ресвҮT [epoc

]ч N20үм MaY[

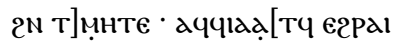

5

$x$ ]е тесгіме еү[тоN

]тбaeiô NTto[

$\pi \times 0 \epsilon] \mathrm{lC} \cdot \pi \in X \lambda y \mathrm{~N}[\mathrm{daC}$

]Ṭ ON NTTINa[

a] $\lambda \lambda d$ XIN TEN[OY

10

е़िмове

1 Hall om. || 2 меп]ресвүт[ерос | меп]ресвүт[ерос Hall || 3 ]ч мгоүм маү[ | ]н м̄доүм ма[ Hall || 4

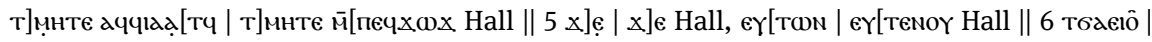

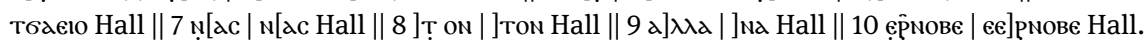

Basierend auf der Edition von Hall hat von Lemm eine ergänzte Textfassung erstellt, bei der er aufgrund des Griechischen und Bohairischen versuchte, einen Text zu rekonstruieren, der im Sahidischen grammatikalisch und inhaltlich korrekt dem Inhalt der Verse der pericope adulterae entspricht. Philologisch sind von Lemms Ergänzungen nach seinem Kenntnisstand gut begründet, nachvollziehbar und korrekt. Um anhand der vorangestellten Reedition zu einer Neubewertung und so zu einer revidierten Textfassung mit durch Neulesungen begründetem Ergebnis zu gelangen, sei von Lemms ergänzter Text Grundlage des Kommentars. Im Verhältnis zur Neulesung gebotene Angaben werden nachstehend als neue Textfassung geboten:

0 [a)antorel]

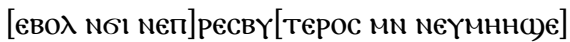

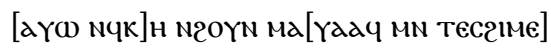

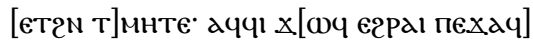
[nac $X]$ ] тесгіме e[YTONN Nen]

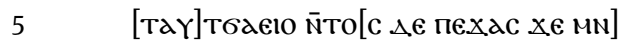

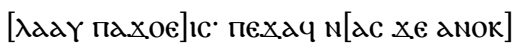

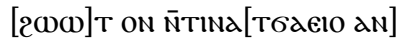
[вок] ме? XIN тем[оY мпркоте] [ер̄] пове 


\section{Kommentar}

0-1 Nach vorhandenen Schriftresten sind Verbesserungen nicht möglich. Die Spuren in Zeile 0 (siehe Zeile 1 der Neuedition), die Hall nicht verzeichnet, konnte von Lemm nicht in die Bearbeitung einbeziehen. Der dem erhaltenen vorangehende Text war umfangreicher als von Lemms Ergänzung ausweisen kann.

$2 \mathrm{~N} \varphi \mathrm{k}]_{\mathrm{H}}$ ist nicht möglich, denn $\mathrm{H}$ ist eine Fehllesung. Korrekt ist $\mathrm{\varphi}$, siehe die Formen in Zeile 3, was aүkad] ч nгоүм ma[rady als Text wahrscheinlich macht und besser griechi-

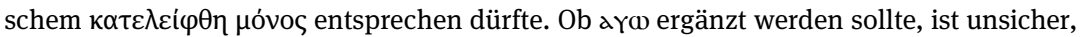
da die Zahl ausgefallener Buchstaben unsicher ist. Es erscheint ferner nicht sinnvoll, wie einige griechische Handschriften ò 'Inбoũ ̧ zu ergänzen, obwohl es bohairisch belegt ist.

3 аччı $x[0 \%$ ist nach erhaltenen Schriftresten unmöglich, da sie nicht zu einem $x$ gehören können. Auch Halls Lesung eines $\mathrm{m}$ anstatt $x$ überzeugt nicht Der Ansatz ähnelt zwar $M$ wie in Zeile 2, doch siehe die Formen von a in Zeile 5-7. Sinnvoller erscheint es nach den erhaltenen Schriftspuren, дччıдатч егpal anzunehmen. Dies würde griechischem

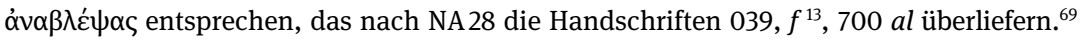
Die Formen aччіеіатч, аччідтч und аччідатч kommen in literarischen Handschriften gleichbedeutend nebeneinander vor. Wahrscheinlich ist nach dem Griechischen ó 'Inбoṽৎ zu ergänzen, doch ist fraglich, ob auch für $\delta \varepsilon ́$ genügend Platz in der Lacuna vorhanden ist. Insgesamt ist wohl aчqıаd Ty ępaı noı ic wahrscheinlich. So ist der Text recht lang und nexay deshalb sicher nicht komplett in diese Zeile zu setzen.

4-5 Gemessen an den umgebenden Zeilen ist von Lemms Ergänzung Nen|Taץ- zu kurz.

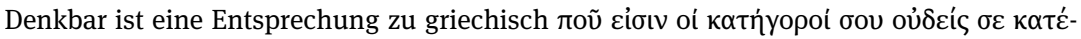

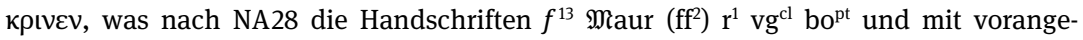

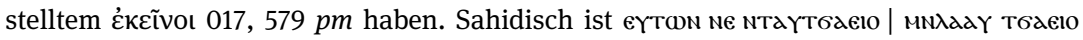
denkbar.

6 Grundsätzlich ist eine koptische Entsprechung für kúpıoৎ zu erwarten. Die vorgeschlagene Ergänzung von пахоєıc darf als unwahrscheinlich angesehen werden; eher ist der einfache Artikel (an Stelle des Possessivartikels) zu vermuten, denn nur einige bohairische Kodizes haben ihn. Keine griechische Handschrift bietet ein Possessivpronomen.

7 Anhand der vorhandenen Schriftreste sind Verbesserungen nicht möglich.

8 Der erhaltene Zeilenbeginn ist klar als ] $\lambda \lambda d$ zu lesen, sodass nd nach Hall und $\mathrm{we}^{?}$ nach von Lemm nicht möglich sind. Es ist kaum denkbar, etwas anderes als d $\lambda \lambda d$ zu ergänzen, obwohl $\dot{\alpha} \lambda \lambda \dot{\alpha}$ griechisch nicht belegt ist.

9 Die Klammersetzung durch von Lemm ist unnötig, da ệpিмовє sicher lesbar ist.

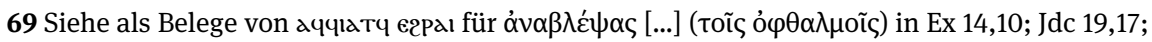
Mt 14,19; Mc 6,41; L 9,16; L 19,5; L 21,1 und für andere griechische Vorlagen und grammatika-

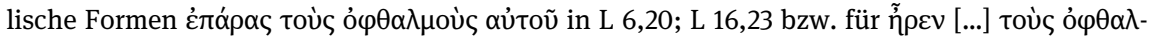

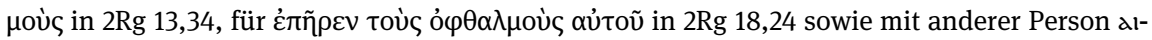

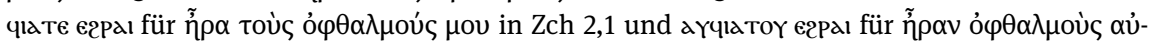

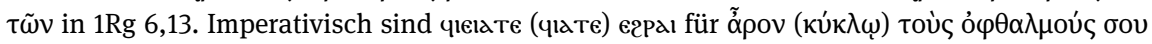

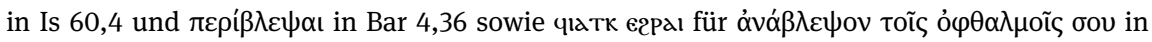

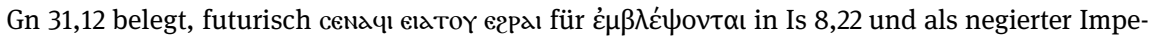

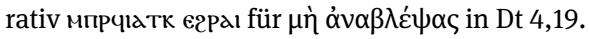


Revidierte Textfassung:

1

$$
\text { ]. [ [ } 9 \text { adantorel }
$$

ево入 NOI Neп]Ресвү[терос мм меүмнно)

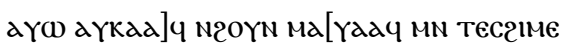

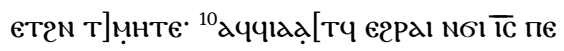

5 Хay nac $x$ ]

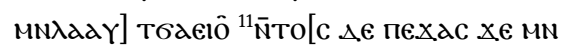

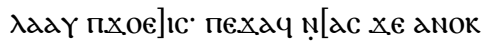
200]] T़ ON N̄TINa[TCAEIO aN вळK a] $\lambda \lambda d$ XIN TEN[OY мпркоте ẹṕnовє 